\title{
Unusually prominent hardware in patellar fracture- a case report
}

\author{
Dr Aakash Mugalur ${ }^{1}$, Dr Binoti A Sheth ${ }^{2}$, Dr Aditya C Pathak ${ }^{3}$,Dr Abhijit Kale ${ }^{4}$ \\ 1(Speciality Medical Officer, Dept. of Orthopaedics, Dr RN Cooper Hospital, Mumbai, India ) \\ 2(Associate Professor \& Unit Chief, Dept. of Orthopaedics, LTMMC \&LTMGH, Mumbai, India) \\ 3(Assistant Professor, Dept. of Orthopaedics, Dr RN Cooper Hospital, Mumbai, India) \\ 4(Assistant Professor, Dept. of Orthopaedics, LTMMC \&LTMGH, Mumbai, India)
}

\begin{abstract}
Patella fractures account for approximately one percent of all skeletal fractures. Traditional methods of fixation include tension-band wiring with heavy gauge wire. Hardware prominence due to the subcutaneous location of patella and operative technique can cause soft-tissue issues and some studies have reported wire breakage or migration, either of which can lead to problems. We report a case of unusual prominence of hardware where the $K$ wires were projecting out of the skin since eight years in a case of healed patella fracture with no evidence of infection and minimal functional disability despite the patient being a diabetic. Utmost attention to the operative technique during the primary surgery and use of newer modalities of treatment like fibre cables can reduce the burden of symptomatic hardware.
\end{abstract}

Keywords: complication, fracture, hardware, implant, patella

\section{Introduction}

Patella, the largest sesamoid bone provides the mechanical advantage and leverage that increases the force of knee extension. Fractures of the patella account for approximately $1 \%$ of all skeletal fractures. Surgical treatment methods include lag screws, Kirshner wires, tension band fixation with wires or non-absorbable sutures with or without screws, and cerclage wiring or a combination of the above.The highest load to failure was seen with the modified tension band and the cannulated lag screw technique. Modified tension band wiring remains a favourable option in the treatment of patellar fractures till date. Owing to the subcutaneous location of the bone there is increased incidence of implant related complications in patellar fractures. We report a case of unusual prominence of hardware where the $\mathrm{K}$ wires were projecting out of the skin since eight years in a case of healed patella fracture with no evidence of infection and minimal functional disability despite the patient being a diabetic.

\section{Case report}

A $70 \mathrm{yr}$ old lady presented to the hospital with low back pain since a month with no history of trauma. The patient had complaints of two Kirschners' wires projecting out of the left knee with occasional anterior knee pain and difficulty of squatting and sitting cross legged. Surprisingly this was neglected for the past eight years. The patient had history of patella fracture treated twelve years back by open reduction and internal fixation. The patient was a known diabetic on treatment.

On clinical examination, apart from signs consistent with low back pain without radiculopathy, we found two $\mathrm{K}$ wires projecting from inferior aspect of patella of the left knee. There was minimal tenderness at the site of projecting $\mathrm{K}$ wires. There was skin pigmentation around the projecting $\mathrm{K}$ wires. There was restriction of last twenty degrees of extension of knee joint on active and passive testing with termimal flexion of knee being painful. The quadriceps were wasted. On radiographs the patella fracture was united with $\mathrm{K}$ wire $-\mathrm{TBW}$ \& broken cerclage wires in situ.( Fig I) Patient was treated by implant removal on an elective basis. The tracts of the $\mathrm{K}$ wires were debrided and closure was achieved.(Fig II) Full extension was achieved intra-operatively by gentle manipulation. Post operatively the wounds healed uneventfully. Patient regained full range of motion and full active extension after supervised physiotherapy and quadriceps strengthening exercises.

\section{Discussion}

Although skin irritation and prominent hardware is common in patellar fractures we did not come across any reported case in literature with unusually prominent $\mathrm{K}$ wires projecting out of the skin. Surprisingly the K-wires were projecting out of the skin since the past eight years and was of minimal concern to the patient. Despite the patient being a diabetic there was no evidence of infection. The healed and contracted skin around the $\mathrm{K}$ wires may have acted as a natural barrier for the infection. The patient also regularly cleaned the $\mathrm{K}$ wires on a daily basis at the time of bath. No other special precaution was taken for the projecting $\mathrm{K}$ wires. Another fact which draws attention in this rare case is the apathy of the patient towards the projecting $\mathrm{K}$ wires for the past eight years. The minimal functional disability and the decreased functional demands at seventy years of age 
might have been responsible for this gross neglect. In spite of the projecting $\mathrm{K}$ wires, clinically there was just restriction of terminal extension with extensor lag and terminal flexion being painful. The degenerative changes of osteoarthritis evident on the radiographs may be partially responsible for the same.

The subcutaneous location of patella and the extensor mechanism results in a high incidence of prominent and symptomatic hardware. Symptoms are related to irritation of skin, capsule and patellar tendon by the implants.Symptoms can be severe enough to necessitate implant removal. An alarmingly high $15 \%$ incidence of soft tissue irritation from hardware that necessitated removal was reported in two case series.In a study by Mereddy et al the overall implant removal rate from the patella was $32.8 \%$ and $40 \%$ in patients younger than 60 years. This high rate of implant removal must be discussed with the patient prior to surgery. Implant removal is not a benign procedure and carries a small risk of further morbidity and additional cost. Complications include anaesthetic complications, wound healing problems, neurovascular injury, and refracture. Implant removal surgery also involves time off from work, financial implications to the patient and to the hospital.Though removal of prominent cerclage or K-wires usually alleviates symptoms and can be performed on an elective basis, ${ }^{7}$ there is no known objective method of predicting who will have good relief of symptoms $\left[{ }^{9}\right]$.

Appropriate care must be taken during the primary surgery to bury the $\mathrm{K}$ wires and cut and bend the $\mathrm{K}$ wires both proximally and distally to avoid problems associated with prominent hardware. Due to the complications and local irritation associated with subcutaneous wiring braided suture tension banding has been tried and biomechanical studies have demonstrated that braided polyester suture is $75 \%$ as strong as wire and performed equivalent to cerclage wire with cyclical loading.Chen et al demonstrated equivalent clinical outcomes with wire versus biodegradable tension band fixation of patellar fractures at a mean of two years of follow up.A study by Warda et al concluded that Compressive External Fixation technique is a safe and effective method of treatment of patella fractures with advantages over traditional forms of fixation particularly in cases with a poor soft tissue envelope, salvage situations, and in locations with limited resources. In yet another study by Lefaivre et al modified tension band technique using four $\mathrm{K}$ wires significantly reduced the problems of prominent hardware.

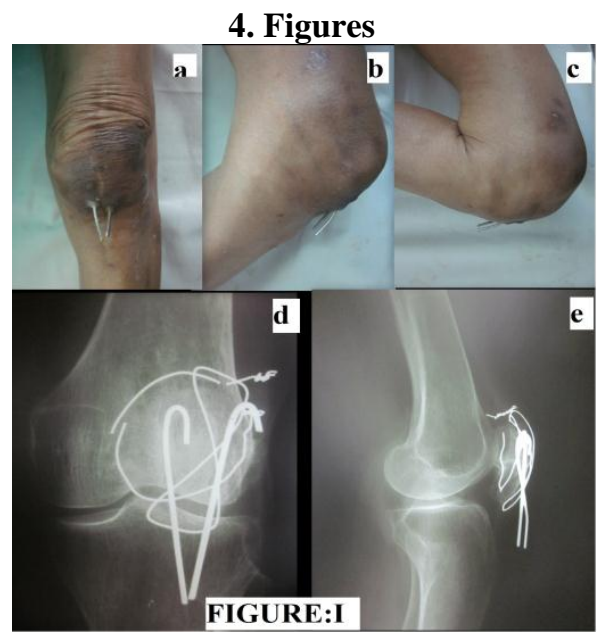

(a, b, c) Clinical photographs showing $\mathrm{K}$ wires projecting out of the skin (d,e) Antero-posterior and lateral radiographs of the knee showing the prominent \& broken hardware

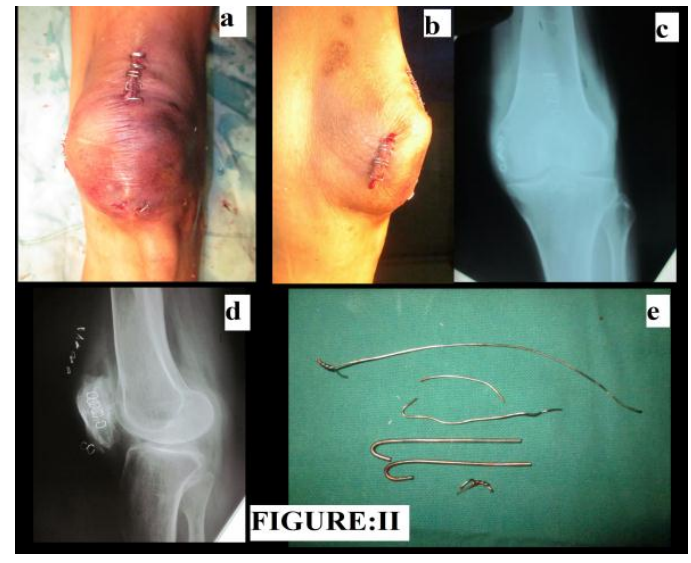


(a,b) Immediate Post operative clinical photographs

(c,d) Antero-posterior and lateral radiographs of the knee after implant removal

(e) Extracted hardware

\section{Conclusion}

Unusually prominent hardware with the $\mathrm{K}$ wires projecting out of the skin since eight years in a case of healed patella fracture with no evidence of infection and minimal functional disability despite the patient being a diabetic is very rare and is unreported in literature. Utmost care and proper attention to the surgical technique at the time of primary fixation can decrease the avoidable problem of symptomatic hardware and complications associated with it. Alternate methods of fixation like partially threaded cannulated screws, non-absorbable sutures, compressive external fixation should be considered in the management of patellar fractures. The high incidence of implant removal should be explained to the patients prior to the surgery.

\section{References:}

[1] Boström A. Fracture of the patella. A study of 422 patellar fractures. Acta Orthop Scand Suppl. 1972; (143):1-80

[2] Anderson LD. Campbell's operative orthopaedics.5th edition. St Louis:Mosby,1971.

[3] Magnuson P.Fractures. 2nd edition. Philadelphia:JB Lippincot,1933.

[4] DePalma A, Flynn J. Joint changes following experimental partial and total patellectomy. JBJS Am 1958;40A:395-413.

[5] Carpenter J, Kasman R, Patel N, et al. Biomechanical evaluation of current patella fracture fixation techniques. J Orthop Trauma $1997 ; 11: 351-356$.

[6] Smith ST, Cramer KE, Karges DE, Watson JT, Moed BR. Early complications in the operative treatment of patella fractures. J Orthop Trauma. 1997; 11(3):183-187.

[7] Bedi A, Karunakar KA, Patella fractures and extensor mechanism injuries: Bucholz RW, Court-Brown CM et al, Rockwood and Green's Fractures in Adults, 7th edition, Lippincott Williams \& Wilkins;2010. p.1752-1779.

[8] Hung L, Chan K, Chow Y et al. Fractured patella:operative treatment using the tension band principle. Injury Br j Accid Surg $1985 ; 16: 343-347$

[9] Mereddy PK, Hakkalamani S. Implant removal following surgical stabilization of patella fracture. Orthopedics. 2010 May 12; 33(5).

[10] McGreal G, Reidy D, Joy A et al. The biomechanical evaluation of polyester as a tension band for the internal fixation of patellar fractures. J Med Eng Technol 1999;23:53-56.

[11] Chen A, Hou C, Bao Jet al. Compression of biodegradable and metallic tension-band fixation for patella fractures. Acta Ortho Scand 1998;69:39-42.

[12] Wardak MI, Siawash AR, Hayda R. Fixation of patella fractures with a minimally invasive tensioned wire method: compressive external fixation. J Trauma Acute Care Surg. 2012 May;72(5):1393-8. 